\title{
Author Correction: Genome-wide association study of classical Hodgkin lymphoma identifies key regulators of disease susceptibility
}

\author{
Amit Sud(10) et al.\#
}

Correction to: Nature Communications; https://doi.org/10.1038/s41467-017-00320-1; published online 1 December 2017

The original version of this Article contained an error in the spelling of a member of the PRACTICAL Consortium, Manuela GagoDominguez, which was incorrectly given as Manuela Gago Dominguez. This has now been corrected in both the PDF and HTML versions of the Article.

Published online: 08 January 2019

\begin{abstract}
(c) (i) Open Access This article is licensed under a Creative Commons Attribution 4.0 International License, which permits use, sharing, adaptation, distribution and reproduction in any medium or format, as long as you give appropriate credit to the original author(s) and the source, provide a link to the Creative Commons license, and indicate if changes were made. The images or other third party material in this article are included in the article's Creative Commons license, unless indicated otherwise in a credit line to the material. If material is not included in the article's Creative Commons license and your intended use is not permitted by statutory regulation or exceeds the permitted use, you will need to obtain permission directly from the copyright holder. To view a copy of this license, visit http://creativecommons.org/licenses/by/4.0/.
\end{abstract}

(C) The Author(s) 2019

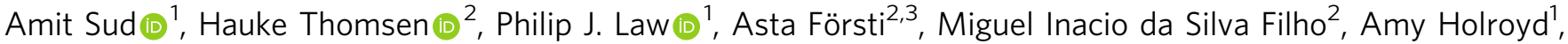
Peter Broderick (1) ', Giulia Orlando ${ }^{1}$, Oleg Lenive ${ }^{1}$, Lauren Wright ${ }^{1}$, Rosie Cooke ${ }^{1}$, Douglas Easton ${ }^{4,5}$, Paul Pharoah (10 ${ }^{4,5}$, Alison Dunning ${ }^{4}$, Julian Peto ${ }^{6}$, Federico Canziann ${ }^{7}$, Rosalind Eeles (10 ${ }^{1,8}$, Zsofia Kote-Jarai ${ }^{1}$, Kenneth Muir (1) 9,10, Nora Pashayan (10,11, The PRACTICAL consortiumPer Hoffmann ${ }^{12,13}$, Markus M. Nöthen ${ }^{13,14}$, Karl-Heinz Jöckel ${ }^{15}$, Elke Pogge von Strandmann ${ }^{16}$, Tracy Lightfoot ${ }^{17}$, Eleanor Kane ${ }^{17}$, Eve Roman ${ }^{17}$, Annette Lake ${ }^{18}$, Dorothy Montgomery ${ }^{18}$, Ruth F. Jarrett ${ }^{18}$, Anthony J. Swerdlow ${ }^{1,19}$, Andreas Engert ${ }^{16}$, Nick Orr ${ }^{20}$, Kari Hemminki ${ }^{2,3}$ \& Richard S. Houlston ${ }^{1,20}$

1Division of Genetics and Epidemiology, The Institute of Cancer Research, London SW7 3RP, UK. Division of Molecular Genetic Epidemiology, German Cancer Research Centre, Heidelberg 69120, Germany. ${ }^{3}$ Centre for Primary Health Care Research, Lund University, Malmö 22100, Sweden. ${ }^{4}$ Centre for Cancer Genetic Epidemiology, Department of Oncology, University of Cambridge, Cambridge CB1 8RN, UK. ${ }^{5}$ Centre for Cancer Genetic Epidemiology, Department of Public Health and Primary Care, University of Cambridge, Cambridge CB1 8RN, UK. ${ }^{6}$ Department of Non-

Communicable Disease Epidemiology, London School of Hygiene and Tropical Medicine, London WC1E 7HT, UK. ${ }^{7}$ Genomic Epidemiology Group, German Cancer Research Center (DKFZ), Heidelberg 69120, Germany. ${ }^{8}$ Royal Marsden NHS Foundation Trust, London SM2 5NG, UK. ${ }^{9}$ Institute of Population Health, University of Manchester, Manchester M13BB, UK. ${ }^{10}$ Division of Health Sciences, Warwick Medical School, Warwick University, 
Warwick CV4 7AL, UK. ${ }^{11}$ Department of Applied Health Research, University College London, London WC1E 7HB, UK. ${ }^{12}$ Department of Biomedicine, Division of Medical Genetics, University of Basel, Basel 4031, Switzerland. ${ }^{13}$ Institute of Human Genetics, University of Bonn, Bonn 53127, Germany. ${ }^{14}$ Department of Genomics, Life \& Brain Center, University of Bonn, Bonn 53127, Germany. ${ }^{15}$ University of Duisburg-Essen, Essen 47057, Germany. ${ }^{16}$ Department of Internal Medicine, University Hospital of Cologne, Cologne 50937, Germany. ${ }^{17}$ Department of Health Sciences, University of York, York Y010 5DD, UK. ${ }^{18}$ MRC University of Glasgow Centre for Virus Research, Glasgow G61 1QH, UK. ${ }^{19}$ Division of Breast Cancer Research, The Institute of Cancer Research, London SW7 3RP, UK. ${ }^{20}$ Division of Molecular Pathology, The Institute of Cancer Research, London SW7 3RP, UK. These authors contributed equally: Amit Sud, Hauke Thomsen. These authors jointly supervised this work: Kari Hemminki, Richard S. Houlston. A full list of consortium members appears at the end of the paper. The original article can be found online at https://doi.org/10.1038/ s41467-017-00320-1.

\section{The PRACTICAL consortium}

Brian E. Henderson ${ }^{21}$, Christopher A. Haiman²1, Sara Benlloch ${ }^{1,5}$, Fredrick R. Schumacher ${ }^{22,23}$, Ali Amin Al Olama ${ }^{5,24}$, Sonja I. Berndt ${ }^{25}$, David V. Conti ${ }^{21}$, Fredrik Wiklund ${ }^{26}$, Stephen Chanock ${ }^{25}$, Victoria L. Stevens ${ }^{27}$, Catherine M. Tangen ${ }^{28}$, Jyotsna Batra ${ }^{29,30}$, Judith Clements ${ }^{29,30}$, Henrik Gronberg ${ }^{26}$, Johanna Schleutker ${ }^{31,32,33}$, Demetrius Albanes ${ }^{25}$, Stephanie Weinstein ${ }^{25}$, Alicja Wolk ${ }^{34}$, Catharine West ${ }^{35}$, Lorelei Mucci $^{36}$, Géraldine Cancel-Tassin ${ }^{37,38}$, Stella Koutros ${ }^{25}$, Karina Dalsgaard Sorensen ${ }^{39,40}$, Lovise Maehle $^{41}$, David E. Neal ${ }^{42,43}$, Ruth C. Travis ${ }^{44}$, Robert J. Hamilton ${ }^{45}$, Sue Ann Ingles ${ }^{21}$, Barry Rosenstein ${ }^{46,47}$, Yong-Jie Lu ${ }^{48}$, Graham G. Giles ${ }^{49,50}$, Adam S. Kibel ${ }^{51}$, Ana Vega ${ }^{52}$, Manolis Kogevinas ${ }^{53,54,55,56}$, Kathryn L. Penney ${ }^{57}$, Jong Y. Park ${ }^{58}$, Janet L. Stanford ${ }^{59,60}$, Cezary Cybulski ${ }^{61}$, Børge G. Nordestgaard ${ }^{62,63}$, Hermann Brenner ${ }^{64,65,66}$, Christiane Maier ${ }^{67}$, Jeri Kim ${ }^{68}$, Esther M. John ${ }^{69,70}$, Manuel R. Teixeira ${ }^{71,72}$, Susan L. Neuhausen ${ }^{73}$, Kim De Ruyck ${ }^{74}$, Azad Razack ${ }^{75}$, Lisa F. Newcomb ${ }^{59,76}$, Davor Lessel $^{77}$, Radka Kaneva ${ }^{78}$, Nawaid Usmani ${ }^{79,80}$, Frank Claessens ${ }^{81}$, Paul A. Townsend ${ }^{82}$, Manuela Gago-Dominguez ${ }^{83,84}$, Monique J. Roobol ${ }^{85}$ \& Florence Menegaux ${ }^{86}$

\footnotetext{
${ }^{21}$ Department of Preventive Medicine, Keck School of Medicine, University of Southern California/Norris Comprehensive Cancer Center, Los Angeles, CA 90033, USA. ${ }^{22}$ Department of Epidemiology and Biostatistics, Case Western Reserve University, Cleveland, OH 44106, USA. ${ }^{23}$ Seidman Cancer Center, University Hospitals, Cleveland, OH 44106, USA. ${ }^{24}$ Department of Clinical Neurosciences, University of Cambridge, Cambridge CB2 1TN, UK. ${ }^{25}$ Division of Cancer Epidemiology and Genetics, National Cancer Institute, NIH, Bethesda, MD 20892, USA. ${ }^{26}$ Department of Medical Epidemiology and Biostatistics, Karolinska Institute, SE-171 77 Stockholm, Sweden. ${ }^{27}$ Epidemiology Research Program, American Cancer Society, 250 Williams Street, Atlanta, Georgia 30303, USA. ${ }^{28}$ SWOG Statistical Center, Fred Hutchinson Cancer Research Center, Seattle, WA 98109, USA. ${ }^{29}$ Australian Prostate Cancer Research Centre-QId, Institute of Health and Biomedical Innovation and School of Biomedical Science, Queensland University of Technology, Brisbane, QLD 4059, Australia. ${ }^{30}$ Translational Research Institute, Brisbane, QLD 4102, Australia. ${ }^{31}$ Department of Medical Biochemistry and Genetics, Institute of Biomedicine, University of Turku, Fl-20520 Turku, Finland. ${ }^{32}$ Tyks Microbiology and Genetics, Department of Medical Genetics, Turku University Hospital, FI-20520 Turku, Finland. ${ }^{33}$ BioMediTech, University of Tampere, Tampere 33100, Finland. ${ }^{34}$ Division of Nutritional Epidemiology, Institute of Environmental Medicine, Karolinska Institutet, SE-171 77 Stockholm, Sweden. ${ }^{35}$ Institute of Cancer Sciences, University of Manchester, Manchester Academic Health Science Centre, Radiotherapy Related Research, The Christie Hospital NHS Foundation Trust, Manchester M13 9NT, UK. ${ }^{36}$ Department of Epidemiology, Harvard School of Public Health, Boston, MA 02115, USA. ${ }^{37}$ CeRePP, Pitie-Salpetriere Hospital, 75013 Paris, France. ${ }^{38}$ UPMC Univ Paris 06, GRC No5 ONCOTYPE-URO, CeRePP, Tenon Hospital, 75020 Paris, France. ${ }^{39}$ Department of Molecular Medicine, Aarhus University Hospital, 8000 Aarhus C, Denmark. ${ }^{40}$ Department of Clinical Medicine, Aarhus University, 8000 Aarhus C, Denmark. ${ }^{41}$ Department of Medical Genetics, Oslo University Hospital, N-0424 Oslo, Norway. ${ }^{42}$ Department of Oncology, Addenbrooke's Hospital, University of Cambridge, Cambridge CB2 0QQ, UK. ${ }^{43}$ Cancer Research UK, Cambridge Research Institute, Li Ka Shing Centre, Cambridge CB2 ORE, UK. ${ }^{44}$ Cancer Epidemiology, Nuffield Department of Population Health, University of Oxford, Oxford OX3 7LF, UK. ${ }^{45}$ Dept. of Surgical Oncology, Princess Margaret Cancer Centre, Toronto, ON M5G 2M9, Canada. ${ }^{46}$ Department of Radiation Oncology, Icahn School of Medicine at Mount Sinai, New York, NY 10029, USA. ${ }^{47}$ Department of Genetics and Genomic Sciences, Icahn School of Medicine at Mount Sinai, New York, NY 10029, USA. ${ }^{48}$ Centre for Molecular Oncology, Barts Cancer Institute, Queen Mary University of London, John Vane Science Centre, London EC1M 6BQ, UK. ${ }^{49}$ Cancer Epidemiology Centre, The Cancer Council Victoria, Melbourne, VIC 3004, Australia. ${ }^{50}$ Centre for Epidemiology and Biostatistics, Melbourne School of Population and Global Health, The University of Melbourne, Melbourne, VIC 3053, Australia. ${ }^{51}$ Division of Urologic Surgery, Brigham and Womens Hospital, Boston, MA 02115, USA. ${ }^{52}$ Fundación Pública Galega de Medicina Xenómica-SERGAS, Grupo de Medicina Xenómica, CIBERER, IDIS, 15782 Santiago de Compostela, Spain. ${ }^{53}$ Centre for Research in Environmental Epidemiology (CREAL), Barcelona Institute for Global Health (ISGlobal), 60803 Barcelona, Spain. ${ }^{54}$ CIBER Epidemiología y Salud Pública (CIBERESP), 28029 Madrid, Spain. ${ }^{55} \mathrm{IMIM}$ (Hospital del Mar Research Institute), 08003 Barcelona, Spain. ${ }^{56}$ Universitat Pompeu Fabra (UPF), 08002 Barcelona, Spain. ${ }^{57}$ Channing Division of Network Medicine, Department of Medicine, Brigham and Women's Hospital/ Harvard Medical School, Boston, MA 02115, USA. ${ }^{58}$ Department of Cancer Epidemiology, Moffitt Cancer Center, Tampa, FL 33612, USA. ${ }^{59}$ Division of Public Health Sciences, Fred Hutchinson Cancer Research Center, Seattle, WA 98109, USA. ${ }^{60}$ Department of Epidemiology, School of Public Health, University of Washington, Seattle, WA 98195, USA. ${ }^{61}$ International Hereditary Cancer Center, Department of Genetics and Pathology, Pomeranian Medical University, 70-001 Szczecin, Poland. ${ }^{62}$ Faculty of Health and Medical Sciences, University of Copenhagen, 1165 Copenhagen, Denmark. ${ }^{63}$ Department of Clinical Biochemistry, Herlev and Gentofte Hospital, University Hospital, 2900 Copenhagen, Denmark. ${ }^{64}$ Division of Clinical Epidemiology and Aging Research, German Cancer Research Center (DKFZ), 69120 Heidelberg, Germany. ${ }^{65}$ German Cancer Consortium (DKTK), German Cancer Research Center (DKFZ), 69120 Heidelberg, Germany. ${ }^{66}$ Division of Preventive Oncology, German Cancer Research Center (DKFZ) and National Center for Tumor Diseases (NCT), 69120 Heidelberg, Germany. ${ }^{67}$ Institute for Human Genetics, University Hospital
} 
Ulm, 89081 Ulm, Germany. ${ }^{68}$ Department of Genitourinary Medical Oncology, The University of Texas M. D. Anderson Cancer Center, Houston, TX 77030, USA. ${ }^{69}$ Cancer Prevention Institute of California, Fremont, CA 94538, USA. ${ }^{70}$ Department of Health Research \& Policy (Epidemiology) and Stanford Cancer Institute, Stanford University School of Medicine, Stanford, CA 94305, USA. ${ }^{71}$ Department of Genetics, Portuguese Oncology Institute of Porto, 4200-072 Porto, Portugal. ${ }^{72}$ Biomedical Sciences Institute (ICBAS), University of Porto, 4200-072 Porto, Portugal. ${ }^{73}$ Department of Population Sciences, Beckman Research Institute of the City of Hope, Duarte, CA 91016, USA. ${ }^{74}$ Faculty of Medicine and Health Sciences, Basic Medical Sciences, Ghent University, 9000 Ghent, Belgium. ${ }^{75}$ Department of Surgery, Faculty of Medicine, University of Malaya, 50603 Kuala Lumpur, Malaysia. ${ }^{76}$ Department of Urology, University of Washington, Seattle, WA 98105, USA. ${ }^{77}$ Institute of Human Genetics, University Medical Center Hamburg-Eppendorf, 20246 Hamburg, Germany. ${ }^{78}$ Molecular Medicine Center, Department of Medical Chemistry and Biochemistry, Medical University, 1431 Sofia, Bulgaria. ${ }^{79}$ Department of Oncology, Cross Cancer Institute, University of Alberta, Edmonton, AB T6G 2R3, Canada. ${ }^{80}$ Division of Radiation Oncology, Cross Cancer Institute, Edmonton, AB T6G 1Z2, Canada. ${ }^{81}$ Molecular Endocrinology Laboratory, Department of Cellular and Molecular Medicine, KU Leuven, 3000 Leuven, Belgium. ${ }^{82}$ Institute of Cancer Sciences, Manchester Cancer Research Centre, University of Manchester, Manchester Academic Health Science Centre, St Mary's Hospital, Manchester M13 9WL, UK. ${ }^{83}$ Genomic Medicine Group, Galician Foundation of Genomic Medicine, Instituto de Investigacion Sanitaria de Santiago de Compostela (IDIS), Complejo Hospitalario Universitario de Santiago, Servicio Galego de Saúde, SERGAS, 15706 Santiago De Compostela, Spain. ${ }^{84}$ University of California San Diego, Moores Cancer Center, La Jolla, CA 92093, USA. ${ }^{85}$ Department of Urology, Erasmus University Medical Center, 3015 CE Rotterdam, The Netherlands. ${ }^{86}$ Cancer \& Environment Group, Center for Research in Epidemiology and Population Health (CESP), INSERM, University Paris-Sud, University Paris-Saclay, F-94805 Villejuif, France 